\section{Estudo dinâmico da mortalidade por tuberculose no Estado de São Paulo, Brasil: uma abordagem bayesiana}

\author{
Dynamic study of tuberculosis mortality in São \\ Paulo State, Brazil: a Bayesian approach
}

1 Departamento de Estatística, Universidade Federal do Amazonas, Manaus, Brasil.

2 Hospital Universitário Clementino Fraga Filho, Universidade Federal do Rio de Janeiro, Rio de Janeiro, Brasil.

Correspondência J. D. O. Santos Junior Departamento de Estatística Universidade Federal do Amazonas.

Rua Dom Emanuel Gomes 37, apto. 202, Rio de Janeiro, $R J$ 21940-350, Brasil.

james@ufam.edu.br

\section{Abstract}

This study used dynamic linear models to analyze the dynamics of tuberculosis (TB) mortality in São Paulo State, Brazil. The study confirms previous findings of a decline in TB mortality in State in the last decade, while pointing to an increase in deaths beginning in 2005. Analysis of the two most frequent underlying causes pointed to HIV/TB co-infection. Forecasts for the next two years were performed, providing little evidence to support a continuing downward trend in the number of TB-related deaths.

Tuberculosis; Mortality; Validity of Tests; Reproducibility of Tests
James Dean Oliveira dos Santos Junior 1 Basílio de Bragança Pereira ${ }^{2}$

\section{Introdução}

Em razão de diversos sintomas, a tuberculose (TB) só foi identificada como doença na segunda metade do século XIX. Embora exista cura, ela ainda aflige a humanidade, permanecendo um problema de saúde pública, particularmente em países em desenvolvimento. Mesmo que houvesse a perspectiva de que a doença poderia ser erradicada, a partir da década de 90, ocorreu um aumento significativo de casos por conta da coinfecção com o HIV. Em 1993, a Organização Mundial da Saúde (OMS) declarou estado de emergência global.

No Brasil, em 2006, a incidência de TB foi estimada em 50 casos por 100 mil habitantes, enquanto a prevalência foi estimada em 55 casos por 100 mil habitantes. O Relatório Global de Controle da Tuberculose de 20081 coloca o Brasil na 16a posição entre os países com maior incidência da doença. Além do advento da AIDS, outros fatores contribuem para o agravamento do quadro da TB no Brasil, como o empobrecimento da população e o sucateamento da Rede Pública de Assistência à Saúde 2 . Outro agravante tem sido o elevado percentual de abandono do tratamento da TB que, nos últimos vinte anos, tem-se mantido em torno de $14 \% 3$. O abandono do tratamento pode levar ao desenvolvimento da TB multirresistente, associada à elevada taxa de mortalidade ${ }^{4}$. Os altos níveis de TB multirresistente detectados no estudo realizado por Cunha 
et al. 5 indicam a falta de medidas públicas para o controle de TB no Brasil.

O estudo da dinâmica da mortalidade de determinada doença constitui ferramenta epidemiológica fundamental, servindo para estudar quantitativamente o comportamento da doença na população e avaliar as intervenções. Para um estudo dinâmico, modelos de previsão são indispensáveis. No Brasil, uma série de estudos foi realizada com propósito de entender a dinâmica da mortalidade por TB. Em Bierrenbach et al. ${ }^{6}$, os autores realizaram um estudo descritivo das taxas de mortalidade anuais para cada estado, cobrindo os anos entre 1984 e 2004. Outro estudo descritivo pode ser visto em Hino et al. 7, no qual foram examinadas as taxas de mortalidade entre os anos de 1980 a 2001. Um estudo interessante utilizando análise de séries temporais foi realizado por Antunes \& Wadlmam 8, no qual os autores analisaram a série de taxas de mortalidade para a cidade de São Paulo. No último, modelos autorregressivos de média móvel (ARMA) foram utilizados.

O presente estudo objetiva examinar as mudanças ocorridas na mortalidade bruta ao longo do tempo para o Estado de São Paulo, utilizando modelos lineares dinâmicos. Tal abordagem possui a vantagem de os parâmetros - como a média - serem variáveis aleatórias, podendo avaliar as mudanças ao longo do tempo. Além disso, esses modelos possuem a propriedade de se adaptar rapidamente às mudanças na série temporal analisada. Este tipo de modelagem já foi utilizado, por exemplo, para prever o número de casos novos de AIDS no Brasil 9 e para previsão de resultados de partidas de futebol 10. Na seção $A$ Série Temporal do Número de Óbitos por Tuberculose no Estado de São Paulo, discutimos a escolha dos dados no estudo. Na seção Modelos Lineares Dinâmicos, apresentamos a metodologia utilizada e a escolha do modelo apropriado. Na seção Resultados, discutimos os resultados obtidos.

\section{A série temporal do número de óbitos por tuberculose no Estado de São Paulo}

Os dados sobre mortalidade por estado podem ser obtidos online pelo Sistema de Informações sobre Mortalidade (SIM. http://www.datasus. gov.br). O SIM foi criado em 1975 pelo Ministério da Saúde para prover estatísticas sobre mortalidade e indicadores de saúde. Até este presente trabalho, os dados disponíveis datavam entre os anos de 1979 e 2006.

Neste trabalho, optamos por utilizar os dados referentes ao Estado de São Paulo. A motivação da escolha advém dos estudos sobre esse estado realizados anteriormente. Por exemplo, em Antunes \& Waldam 8 , um grande esforço foi empreendido para reunir informações sobre a mortalidade por TB entre nos anos de 1900 a 1997, a fim de realizar uma análise temporal estatística utilizando a classe de modelos ARMA. Além disso, os resultados em Antunes \& Waldam 8 concordam com um estudo descritivo mais recente, feito em Bierrenbach et al. 6, no qual as taxas de mortalidade para o Estado de São Paulo apresentam declínio até o fim do ano de 2004.

Preferimos também utilizar dados mensais, pois nesse nível de detalhamento podemos caracterizar o efeito sazonal da série - tal efeito não pode ser observado com a série anual. Resolvemos, também, estudar a dinâmica do número total de óbitos registrados por mês, em vez das taxas de mortalidade. Com isso, podemos prever quantitativamente o número de óbitos em um dado horizonte no tempo.

Por causa da mudança da 9a Classificação Internacional de Doenças (CID-9) para CID-10, utilizaram-se os dados a partir de 1996. Os códigos de causa do óbito foram utilizados seguindo Bierrenbach et al. 6: (1) TB pulmonar (CID-9: 100, 109-119; CID-10: A150-A153. A160-A162 e A169); (2) TB respiratória extrapulmonar (CID-9: 101108 120-129; CID-10: A154-A159, A163-A168); (3) TB extrapulmonar (CID-9: 130-189; CID-10: A170-A179); (4) TB congênita (CID-10: P370); (5) sequelas de TB (CID-10: B90); (6) doença pelo HIV resultando em TB (CID-10: B20).

Entre os anos de 1996 e 2006, a TB pulmonar e a doença por HIV resultando por TB foram responsáveis por 97,23\% dos óbitos (por TB) registrados.

\section{Modelos lineares dinâmicos}

Os modelos de previsão bayesianos que utilizam modelos lineares dinâmicos foram introduzidos em Harrison \& Stevens 11. A abordagem é fundamentalmente composta por: (1) uma definição de um modelo sequencial; (2) estruturação de modelos paramétricos com parametrização significativa para o modelador; (3) representação probabilística da informação sobre os parâmetros; (4) previsões calculadas com base numa distribuição de probabilidade.

Um caso particular dentre os modelos de previsão bayesianos é modelo linear dinâmico, baseado na distribuição normal. Essa escolha é bastante razoável, já que grande parte dos modelos estatísticos de previsão é baseada na suposição de normalidade. Além disso, a distribuição normal possui diversas vantagens, como fácil conjugação e uma distribuição multivariada fá- 
cil de lidar. O modelo linear dinâmico permite incorporar componentes de tendências e sazonalidade, permitindo predições adequadas ao comportamento dos dados. Neste trabalho, utilizamos a teoria de modelos lineares dinâmicos para analisar a dinâmica do número de óbitos por TB. Para a análise, usamos os seguintes componentes:

a) Tendência linear: componente que dá a direção das previsões futuras, baseada na informação corrente. Ela é dividida em duas partes - nível e tendência. O nível representa o intercepto da previsão, ou seja, a média atual das observações, desconsiderando outras componentes. A tendência informa sobre o crescimento/declínio dos valores futuros da média dos dados. Assim, uma tendência positiva (negativa) implica crescimento (declínio) da média. Uma tendência próxima de zero implica que as previsões futuras estarão próximas do nível atual dos dados, a menos de outras componentes.

b) Efeito sazonal: componente que controla o efeito sazonal dos dados. Um efeito sazonal é um comportamento que se repete após um período de tempo (como as estações do ano, por exemplo). Existem duas formas usuais para caracterizar as oscilações do efeito sazonal. Utilizaremos a sazonalidade trigonométrica, que decompõe o efeito sazonal em funções de cossenos. Cada uma dessas funções é denominada harmônico.

Com a modelagem dinâmica, pode-se obter: a) Estimativas suavizadas das componentes: essa técnica utiliza toda a série temporal para estimar novamente as componentes do modelo. A ideia básica sobre esse tipo de técnica é que não só o passado e o presente ajudam a prever o futuro, mas também a nossa compreensão do presente nos auxilia a entender o passado.

b) Previsões: as previsões podem ser feitas tanto para as componentes quanto para as observações futuras. Como esperado, ao tentar prever um horizonte longe no tempo, a precisão das previsões fica comprometida. Portanto, previsões em curto prazo sempre serão mais precisas e podem ser utilizadas para verificar se o modelo é adequado aos dados, como será mostrado na próxima seção.

O modelo linear dinâmico é apresentado formalmente na Figura 1. Para uma leitura complementar, sugerimos Pole \& Harrison 12 e Petris et al. 13 .

\section{Resultados}

Analisamos o número total de óbitos registrados por mês que tiveram por causa básica a TB. A análise também foi realizada, em separado, para os dois tipos de causas básicas mais frequentes: TB pulmonar e doença por HIV seguido de infecção por TB.

O modelo utilizado foi o linear dinâmico com tendência linear e sazonalidade trigonométrica a escolha desse modelo está justificada em nota, na Figura 1. A Figura 2 mostra o número total de óbitos registrados e o previsto para cada mês. Pode-se ver que os valores previstos para cada mês se aproximam do valor observado com o passar dos meses. Note ainda que a maioria dos pontos observados está no intervalo de predição com 90\% de credibilidade, o que sugere a adequação do modelo para previsões em curto prazo.

Na Figura 3, mostram-se as estimativas suavizadas para o nível e a tendência. Podemos constatar que a tendência permaneceu abaixo de zero entre os anos de 1996 e 2004. Isso caracteriza um período com tendência ao declínio do número total de óbitos registrados por mês. Nesse mesmo período, o nível médio da série também decresceu. Tais resultados reforçam a análise descritiva dada em Bierrenbach et al. ${ }^{6}$. Entretanto, a partir de 2005, a tendência voltou a crescer, assim como o seu nível. Em 2006, a tendência se estabilizou em torno de um, enquanto o nível da série continuou a crescer (Figura 3, tendência).

As estimativas suavizadas para a sazonalidade também são mostradas na Figura 3. Os dados sugerem que o efeito sazonal é nulo nos meses de abril e outubro. Um efeito positivo, i.e, um aumento no número total de óbitos é encontrado nos meses maio, junho, julho, agosto e setembro. Um efeito negativo (diminuição do número total de óbitos) ocorre no restante dos meses.

As previsões para os anos de 2007 e 2008 são mostradas na Figura 4. Pode-se notar que, ainda que exista uma expectativa de aumento do número total de óbitos por mês para a TB, os limites de credibilidade permitem um cenário otimista, caracterizado pelo limite inferior do intervalo de $90 \%$ de credibilidade, tendo resultados semelhantes aos de 2004 e 2005. Entretanto, o cenário esperado é o crescimento do nível dessa série, mantendo o padrão de 2006 (ou aumentando). É importante ressaltar que um modelo dinâmico tende a levar em consideração os dados mais recentes, logo as previsões estão mais relacionadas com os dados de 2005 e 2006 que os demais anos.

Como discutido anteriormente, mais de $97 \%$ dos óbitos por TB registrados entre 1996 e 2006 foram ou por TB pulmonar ou por coinfecção HIV/TB. Por esse motivo, torna-se interessante analisar separadamente ambas. O mesmo modelo foi aplicado para as séries temporais das duas causas básicas de óbito citadas. As estimativas suavizadas para o nível e tendência de ambas 
Modelo linear dinâmico utilizado.

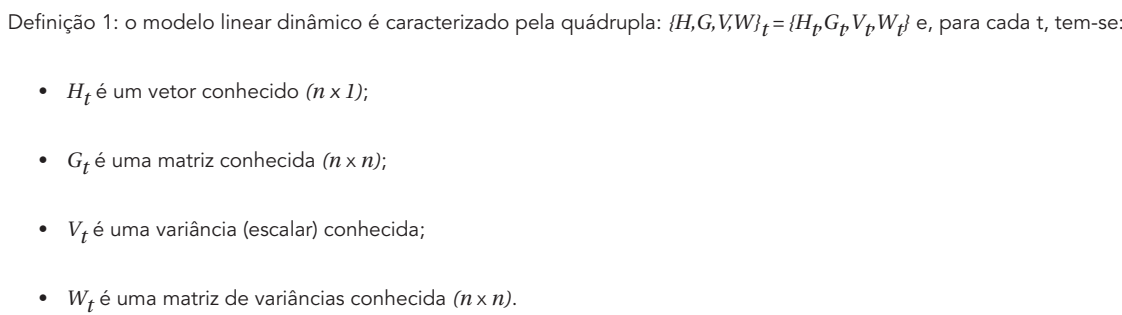

Esta quádrupla define o modelo relacionando $Z_{t}$ ao vetor de parâmetros $\theta_{t}(n \times 1)$ no tempo t, e a evolução de $\theta_{t}$ através do tempo, via a especificação das distribuições:

$\left(Z_{t} \mid \theta_{t}\right) \sim N\left(H_{t}^{T} \theta_{t}, V_{t}\right)$

$\left(\theta_{t} \mid \theta_{t-1}\right) \sim N\left(G_{t}^{T} \theta_{t-1}, W_{t}\right)$

Com a escolha certa para $H_{t}$ e $G_{t}$, pode-se construir a maioria das funções de previsão da literatura. Os seguintes casos serão usados neste trabalho:

- Modelo de tendência polinomial de primeira ordem:

- Modelo de tendência polinomial de segunda ordem (ou de tendência linear):

$H_{t}=\left(\begin{array}{l}1 \\ 0\end{array}\right), G_{t}=\left(\begin{array}{ll}1 & 1 \\ 0 & 1\end{array}\right)$

- Modelo sazonal com componentes harmônicas de período p (par):

$$
H_{t}=\left(\begin{array}{c}
E_{2} \\
E_{2} \\
\vdots \\
E_{2} \\
1^{T}
\end{array}\right), G_{t}=\left(\begin{array}{cccccc}
J_{2}(1, \omega) & 0 & \cdots & 0 & 0 & \\
0 & J_{2}(1, \omega) & \ldots & 0 & 0 & \\
\vdots & \vdots & \ddots & \vdots & \vdots \\
0 & 0 & \cdots & J_{2}(1, h \omega-\omega) & 0 \\
0 & 0 & \cdots & 0 & -1
\end{array}\right)
$$

onde $E_{2}=(1,0, \ldots, 0), 1^{T}=(1,1, \ldots, 1), \omega=2 \pi / p$ e

$J_{2}(1, \omega)=\left(\begin{array}{cc}\cos (\omega) & \sin (\omega) \\ -\sin (\omega) & \cos (\omega)\end{array}\right)$

Esses modelos podem ser superpostos (West \& Harisson 14, Teorema 6.4., p. 187). As previsões um passo à frente serão realizadas através da função de previsão.

$$
f_{t}=E\left(Z_{t+1} \mid D_{t}\right)=H_{t+1}^{T} G_{t+1} m
$$

Nota: Por simplicidade, denotaremos os modelos por $\operatorname{Mod}(0, h)$, onde o $(>0)$ é a ordem da componente polinomial e $h$ é a quantidade de harmônicos utilizada ( $h=0$ implica que o modelo não possui a parte sazonal). A priori, foi assumido que a média do nível era de 140 e a média das outras componentes foi tomada como nula. A variância dos termos foi tomada como 900 (ou seja, a priori os dados estão entre $140 \pm 60$ com probabilidade aproximada de 95\%). A variância Wt foi estimada com base na função de verossimilhança (Petris et al. 13). A Figura 6 mostra a raiz do erro quadrático médio (EQM) e o erro médio absoluto (EAM) para previsões realizadas pelos melhores modelos (o cálculo dessas medidas foi realizado retirando as 12 primeiras observações, pois elas são altamente influenciadas pela priori). Utilizando o critério de minimização dos erros, optamos pelo modelo $\operatorname{Mod}(2,1)$, ou seja, pelo modelo de tendência linear (segunda ordem) com um harmônico.

estão na Figura 5. É importante notar que, para o número total de óbitos por TB respiratória, tanto o nível quanto a tendência decresceram ao longo dos anos. Para os anos de 2005 e 2006, podemos verificar que o nível aparenta certa estabilidade (70 óbitos por mês). Além disso, a ten- dência para esses mesmos anos está próxima de zero, o que implica previsões para os próximos meses em torno do nível médio 70 óbitos por mês. Todavia, o mesmo não ocorre com a causa básica coinfecção HIV/TB. Para essa causa, notamos aumento no nível, começando em 2004. 


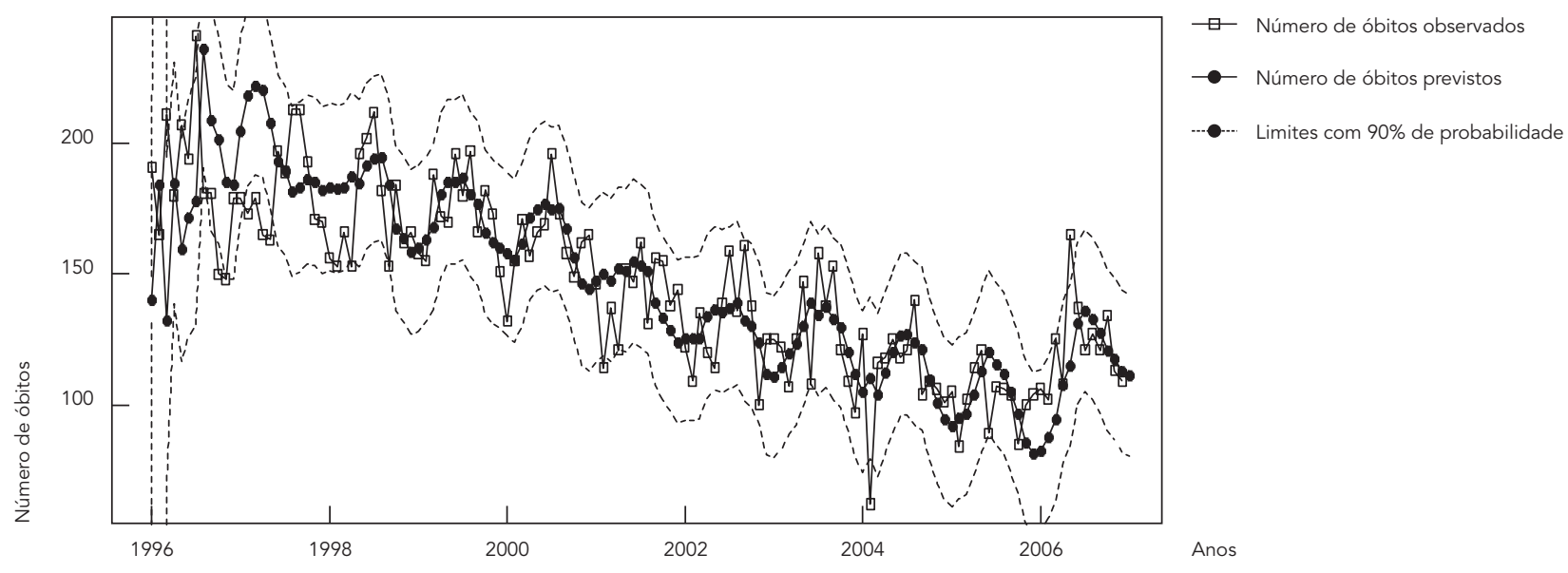

Figura 3

Decomposição da função de previsão em nível, tendência e sazonalidade.

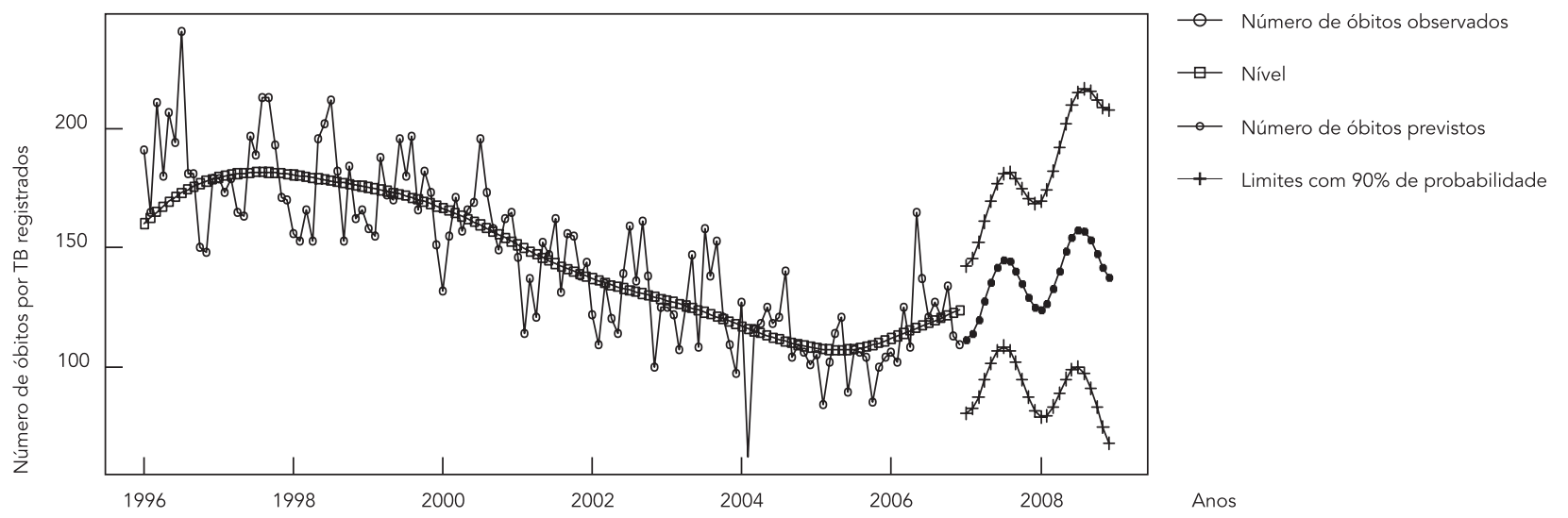

TB: tuberculose

Por volta do fim de 2004, a tendência começou a ficar positiva. Tanto o aumento do nível quanto da tendência indica que o aumento observado na série geral de óbitos por TB registrados é devido a essa causa básica. $O$ cenário atual sugere que o aumento estimado para os anos de $2007 \mathrm{e}$ 2008 para o número de obtidos por TB será por causa da coinfecção HIV/TB.

\section{Considerações finais}

Os modelos lineares dinâmicos se mostraram aptos a recuperar informações já reveladas em estudos descritivos dos dados de mortalidade por TB. A decomposição em nível/tendência/sazonalidade ajuda a entender melhor o comportamento da série, podendo, por exemplo, facilitar a análise 
Figura 4

Série histórica e previsões para o número médio de registros de óbitos por tuberculose para os anos 2007 e 2008.

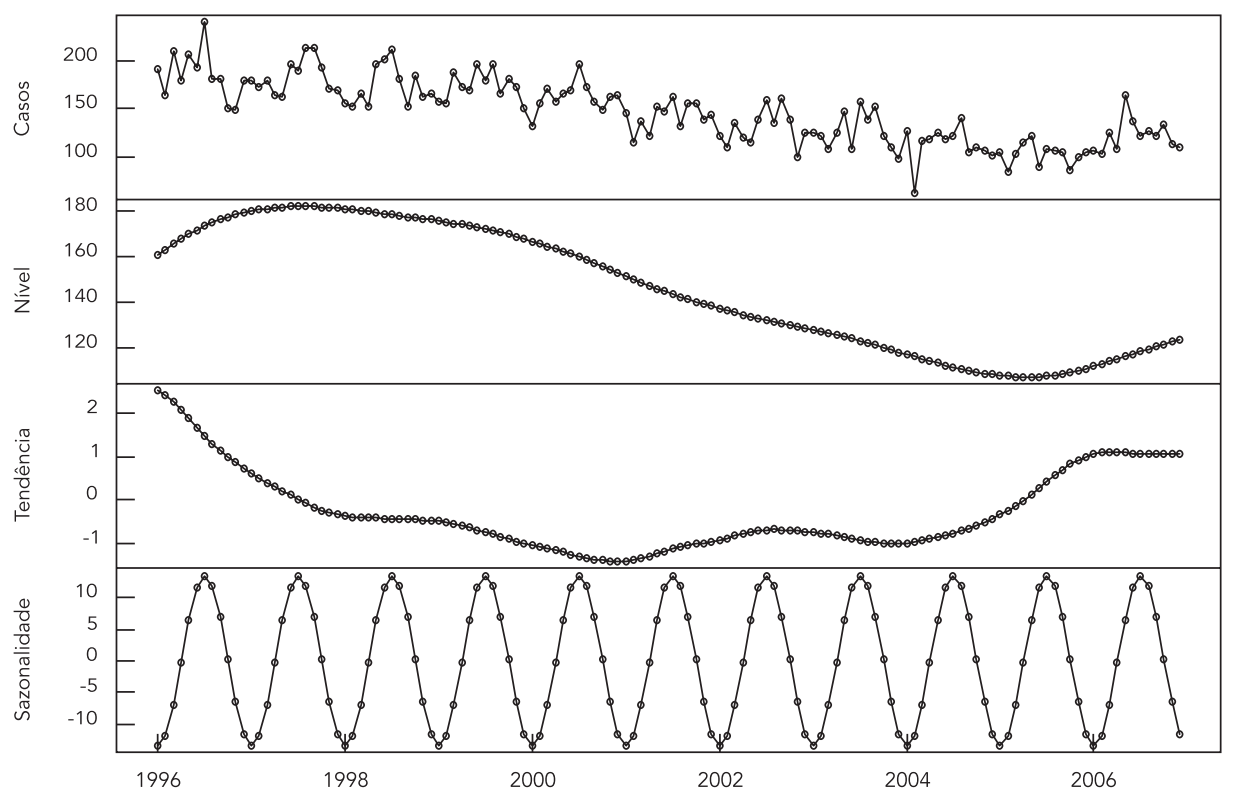

Figura 5

Estimativas suavizadas para o nível e tendência das séries históricas de óbitos por tuberculose (TB) registrados, em que as causas básicas foram TB pulmonar e coinfecção HIV/TB.

5a) TB pulmonar

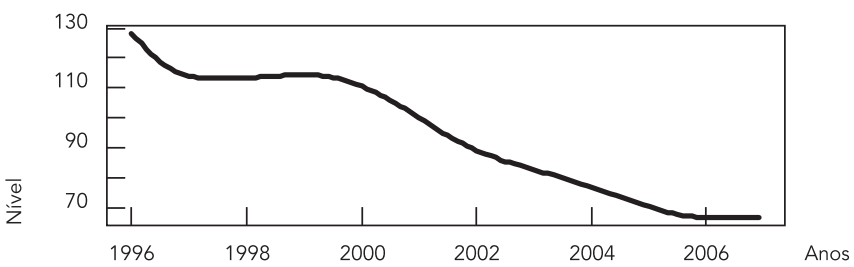

5c) Coinfecção HIV/TB

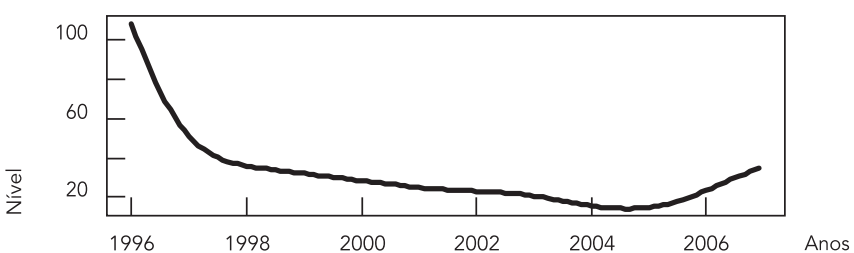

5b) TB pulmonar

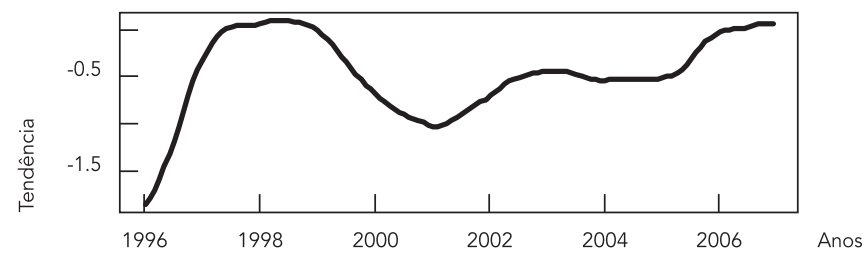

5d) Coinfeccção HIV/TB

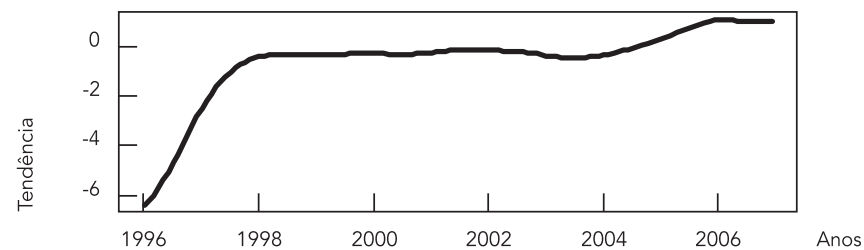


Erro quadrático médio e erro absoluto médio de previsão para os modelos propostos.

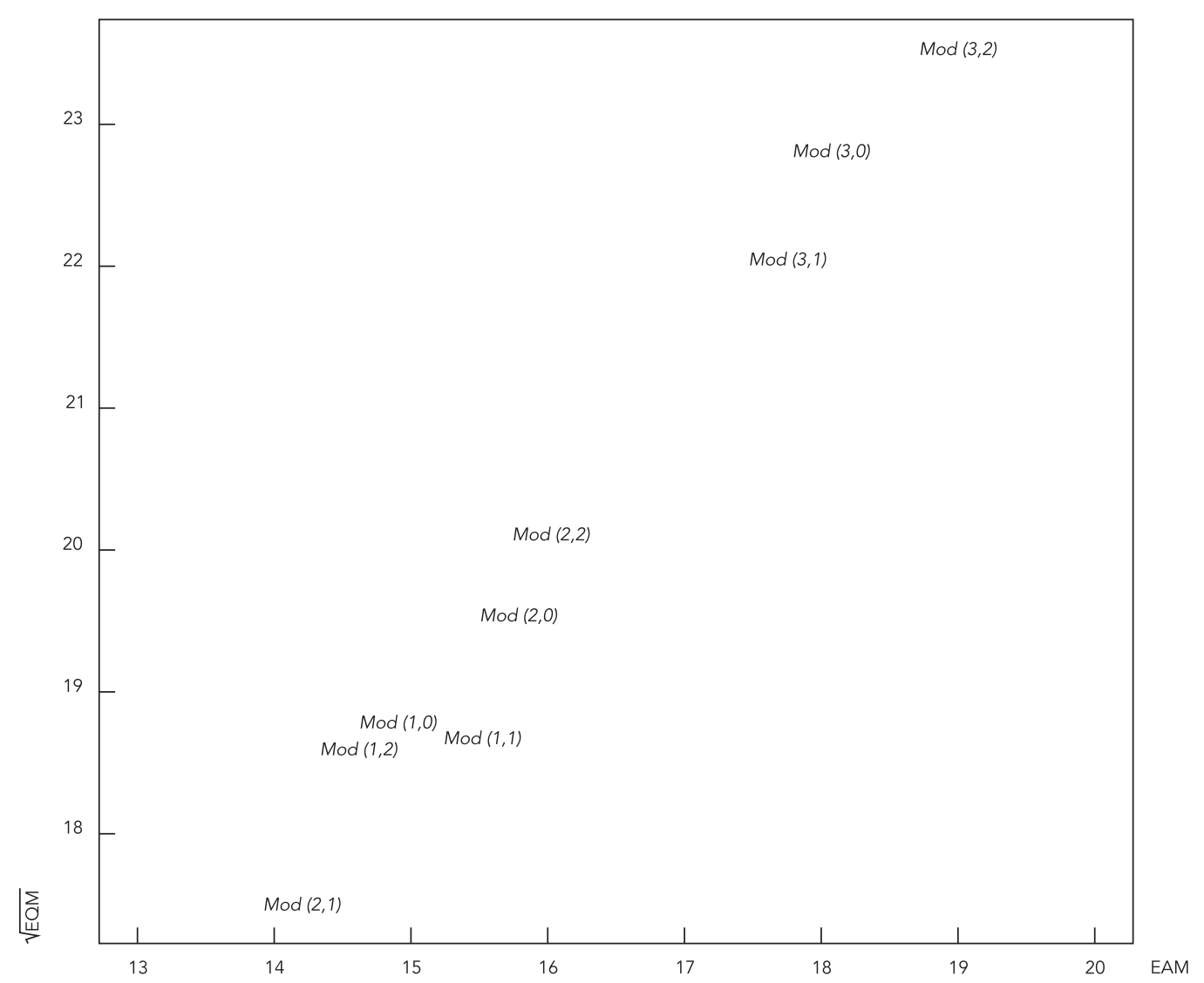

EAM: erro médio absoluto; EQM: erro quadrático médio.

do gestor público sobre a eficácia de uma nova medida de intervenção. Além disso, os modelos dinâmicos lineares permitem que o gestor possa estimar com razoável precisão o comportamento futuro da série, servindo como ferramenta de monitoração/intervenção.

Pela análise obtida neste trabalho, levantaram-se evidências de que o Estado de São Paulo vivenciou um período de baixa no número total de óbitos por TB, concordando com os estudos empíricos citados na seção A Série Temporal do
Número de Óbitos por Tuberculose no Estado de São Paulo. Entretanto, esta mesma análise, para os anos de 2005 e 2006 incluídos, revela um aumento no número total de óbitos. Uma análise mais detalhada que levou em conta apenas as duas causas básicas com maior número de óbitos revelou que tal aumento se deveu à coinfecção HIV/TB. Previsões para 2007 e 2008 indicam que o número total de óbitos por essa causa básica aumentou. 


\section{Resumo}

Neste trabalho, utilizamos a técnica bayesiana de modelos lineares dinâmicos para estudar a dinâmica da mortalidade por tuberculose (TB) no Estado de São Paulo, Brasil. O estudo confirma os resultados empíricos da literatura, que indicam o decaimento da mortalidade para esse estado na última década. Este trabalho também indica um crescimento no número de óbitos a partir de 2005. A análise das duas causas básicas com maior ocorrência revelou que tal aumento é em razão da coinfecção HIV/TB. Previsões para os próximos dois anos foram realizadas, dando poucas evidências para sustentar a continuidade de tendência decrescente do número de óbitos por TB.

Tuberculose; Mortalidade; Validade dos Testes; Reprodutibilidade dos Testes

\section{Colaboradores}

J. D. O. Santos Junior participou da concepção do trabalho, organização dos dados, revisão bibliográfica, análise estatística e elaboração do artigo. B. B. Pereira colaborou na concepção inicial do trabalho, revisão da análise estatística e revisão do trabalho final.

\section{Agradecimentos}

Os autores agradecem o auxílio financeiro recebido pelo Conselho Nacional de Desenvolvimento Científico e Tecnológico (CNPq) e pelo Projeto de Treinamento ICHORTA-AIDS/TB.

\section{Referências}

1. World Health Organization. Global tuberculosis control report 2008. http://www.who.int/tb/pub lications/global_report/2008/update/en/index. html (acessado em 01/Jan/2010).

2. Ruffino Netto A. Doenças emergentes ou reemergentes? Medicina (Ribeirão Preto) 1997; 30:405.

3. Almeida SAD, Honer MR. Abandono do tratamento da tuberculose em duas unidades de referência de Campo Grande, MS - 2002 e 2003. Bol Pneumol Sanit 2006; 14:167-71.

4. Kritski AL. Emergência de tuberculose resistente: renovado desafio. J Bras Pneumol 2010; 36:157-8.

5. Marques M, Cunha EAT, Ruffino-Netto A, Andrade SMO. Drug resistance profile of Mycobacterium tuberculosis in the state of Mato Grosso do Sul, Brazil, 2000-2006. J Bras Pneumol 2010; 36:224-31.

6. Bierrenbach AL, Gomes ABF, Noronha EF, Souza MFM. Tuberculosis incidence and cure rates, Brazil, 2000-2004. Rev Saúde Pública 2007; 41 Suppl 1:24-33.

7. Hino P, Costa-Júnior ML, Sassaki CM, Oliveira MF, Villa TCS, Santos CB. Time series of tuberculosis mortality in Brazil (1980-2001). Rev Latinoam Enferm 2007; 15:936-41.
8. Antunes JLF, Waldman EA. Tuberculosis in the twentieth century: time-series mortality in São Paulo, Brazil. Cad Saúde Pública 1999; 15:463-76.

9. Gamerman D, Migon HS. Forecasting the number of AIDS cases in Brazil. Statistician 1991; 40:427-42.

10. Owen A. Dynamic bayesian forecasting models of football match outcomes with estimation of the evolution variance parameter. IMA Journal of Management Mathematics 2011; 22:99-113.

11. Harrison PJ, Stevens CF. Bayesian forecasting. J R Stat Soc Series B Stat Methodol 1976; 38:205-47.

12. Pole AW, Harrison PJ. Applied bayesian forecasting and time series analysis. New York: Chapman-Hall; 2001.

13. Petris G, Petrone S, Campagnoli P. Dynamic linear models with R. New York: Springer-Verlag; 2010.

14. West M, Harrison, J. Bayesian forecasting and dynamic models. 2nd Ed. New York: Springer-Verlag; 1999.

Recebido em 18/Set/2009

Versão final reapresentada em 01/Mar/2011

Aprovado em 26/Abr/2011 